\title{
Impact of isotretinoin treatment on testosterone and sex hormone- binding globulin in malepatients with acne vulgaris
}

\author{
Original \\ Yasser S. Nasrallah ${ }^{1}$, Halim Maher', Maha M. Anani' ${ }^{2}$ Moustafa M. K. Eyada ${ }^{1}$ \\ Article \\ ${ }^{1}$ Department of Dermatology, Venereology and Andrology, Faculty of Medicine, Suez Canal \\ University, Ismailia, Egypt and Shaqra University, Saudi Arabia, ${ }^{2}$ Department of Clinical \\ Pathology, Faculty of Medicine, Suez Canal University Ismailia, Egypt.
}

\begin{abstract}
Background: Acne vulgaris is primarily a disease of the pilosebaceous unit. Four main pathogenic factors are known to lead to its development: follicular epidermal hyperproliferation, excess sebum production, inflammation, and the activity of propionibacterium acnes. Isotretinoin (ISO) is a 13-cis-retinoic acid derivative of vitamin A. It is a highly effective therapy for severe nodulocystic acne.

Objective: The aim was to measure serum total, free testosterone, and sex hormone-binding globulin (SHBG) levels, before and after treatment, in male patients suffering from acne vulgaris and taking ISO as a treatment for their acne.

Patients and Methods: The authors included 113 male patients with acne vulgaris. The patients received $0.5 \% \mathrm{mg} / \mathrm{kg} / \mathrm{day}$ ISO treatment. Blood samples were collected from the patients for analysis of total, free testosterone, and SHBG levels before and after 6 months of treatment.

Results: After 6 months of treatment with ISO, the total lesion count was significantly lower after treatment $(88.70 \pm 35.04$ and $27.80 \pm 17.94$, respectively, $P<0.05$ ). There was significant reduction in mean total testosterone serum levels after treatment $(5.553 \pm 2.614$ and3.329 \pm 2.276 , respectively, $P<0.05)$. The mean free testosterone serum level was insignificantly lower after treatment (31.237 \pm 11.994 and $24.526 \pm 14.917$, respectively, $P>0.05)$, and the mean SHBG serum level was insignificantly higher after treatment (104.367 \pm 14.689 and105.533 \pm 16.362 , respectively, $P>0.05)$.

Conclusion: This study showed that ISO treatment led to significant reduction in serum total testosterone levels, which may be beneficial for tackling the pathogenesis of acne.
\end{abstract}

Key Words: Acne vulgaris, isotretinoin, serum testosterone, SHBG.

Received: 24 July 2019, Accepted: 09 March 2020

Corresponding Author: Yasser S. Nasrallah, Department of Dermatology, Venereology and Andrology, Faculty of Medicine, Suez Canal University Ismailia, Egypt, Tel.: 01016932575, E-mail: dryassersalem@yahoo.com

ISSN: 2090-6048, September 2019 Vol. 9, No. 3

\section{INTRODUCTION}

Acne vulgaris is primarily a disease of the pilosebaceous unit. Four main pathogenic factors are known to lead to its development including follicular epidermal hyperproliferation, excess sebum production, inflammation, and the presence and activity of Propionibacterium acne; with the onset of puberty, androgen-mediated stimulation of the sebaceous gland results in increased sebum production $^{[1]}$.

Several hormones implicated in the regulation of sebaceous gland activity have been linked to acne.They include androgens, estrogens, growth hormone, insulin, insulin-like growth factor-1, corticotropin-releasing hormone, adrenocorticotropic hormone, melanocortins, and glucocorticoids ${ }^{[2]}$. Individuals who are intrinsically insensitive to androgens do not produce sebum and do not develop acne ${ }^{[3]}$.

The available literature regarding the relationship between androgenicity and acne severity is not consistent in its conclusions. It is still not clear how the hormonal stimulation is related to the clinical expression and the course of the disease.Serum testosterone and sex hormone-binding globulin (SHBG) are common androgens associated with acne $\mathrm{e}^{[4]}$.

Increased level of serum testosterone and decreased level of SHBG have been shown in patients with acne. The evaluation of serum SHBG levels in women with acne is strongly recommended in order to select patients who can have a better response to appropriate hormonal regimes ${ }^{[5]}$.

However, many reports indicate no significant association between acne severity and androgen levels ${ }^{[6]}$.

Isotretinoin (ISO) is a synthetic vitamin A analog and the most effective treatment modality for severe acne. ISO is the only agent which is effective on all of acne pathogenesis steps ${ }^{[7]}$.

There are a lot of different low-dose regimens used 
in the literature, such as daily, intermittent therapy, alternate-day therapy, or gradually increasing the daily dose. In these treatment modalities, the most frequently used dose of ISO is $0.5 \mathrm{mg} / \mathrm{kg} /$ day $^{[8]}$.

It has been found that ISO treatment induces several changes in the hormonal status of acne patients: cortisol, adrenocorticotropic hormone, luteinizing hormone, total testosterone, and prolactin levels decline significantly after 3 months of treatment which may be beneficial for tackling the pathogenesis of acne ${ }^{[9]}$.

This research was conducted to understand more about the effect of ISO treatment on testosterone and SHBG in male patients with acne vulgaris.

\section{PATIENTS AND METHODS}

\section{Type of the study}

This is a quasi-experimental study.

\section{Study location}

The study was carried out in the Dermatology and Andrology Outpatient Clinic of Suez Canal University Hospital.

\section{Study population}

Male patients who have moderate-to-severe acne vulgaris attending the Dermatology Outpatient Clinic of Suez Canal University Hospital at Ismailia city.

\section{Inclusion criteria:}

(1) Sex: male patients.

(2) Age: 16-30-year.

(3) All patients were diagnosed with moderate-tosevere acne.

\section{Exclusion criteria:}

(1) Patients using vitamin A supplements.

(2) Sensitivity or allergy to parabens.

(3) Previously diagnosed thyroid or pituitary disease.

(4) Recent history of psychiatric, mood, or depressive disorders.

(5) Previous therapy with oral retinoids or hormone therapy for any reason in the last 3 months.

(6) Patients with hepatic disease and diabetes mellitus.

\section{All patients were subjected to the following:}

(1) History taking included name, age, occupation, marital status, smoking, and history of using any topical or systemic drugs for the treatment of acne vulgaris, history of allergy, or hypersensitivity drugs.

(2) Dermatological examination of acne:at baseline and one per month for 6 months to observe clinical changes and any side effects or complications and includes size of the lesion, degree of inflammation, and lesion count and to determine the severity of acne vulgaris according to the Lehmann classification as ${ }^{[10]}$ : (a) Mild acne: less than 20 comedones, less than 15 inflammatory lesions, or total lesion count of less than 30 .

(b) Moderate acne: from 20 to 100 comedones, from 15 to 50 inflammatory lesions, or total lesion count from 30 to 125 .

(c) Severe acne: more than 5 cysts, total comedones count of more than 100, total inflammatory count of more than 50 , or total lesion count of more than 125 .

\section{Special biochemical investigations: Blood sampling:}

Blood samples were obtained by venipuncture of the large antecubital veins and were placed in sterile plain tubes and the tube has been centrifuged for $15 \mathrm{~min}$ at $1000 \mathrm{~g}$. Serum has been removed and immediately stored at $20^{\circ} \mathrm{C}$ or $-80^{\circ} \mathrm{C}$. Laboratory investigations were performed at baseline and 4 months after oral ISO use. All samples were studied together on the same day using the same kits.

Serum total and free testosterone concentrations were measured usingthe enzyme-linked fluorescent assay (ELFA) method with VIDAS, BioMerieux company, France. The normal reference value in normal adultmen was $2.8-8.0 \mathrm{ng} / \mathrm{ml}$; levels of free testosterone $(10-40 \mathrm{ng} /$ $\mathrm{ml}$ ) and serum SHBG concentration were measured using SHBG enzyme-linked immunosorbent assay (ELISA) and the normal reference value in normal adultmen was 60 $110 \mu \mathrm{mol} / 1$.

\section{Treatment protocol}

ISO therapy was initiated at a dose of $0.5 \mathrm{mg} / \mathrm{kg}$ body weight. The drug was administered once per day with meals. Treatment was continued for 6 months.

\section{Assessment}

The patients were followed up every month for 6 months and each time the patients were assessed to observe the clinical changes and for any side effects or complications.

Assessment of serum levels of the free and total testosterone

Serum total and free testosterone were measured usingminiVIDAS(Vitek Immunodiagnostic Assay System) through anELFA.The miniVIDAS is equipped with the ELFA technology, making it one of the most economical, efficient, and reliable systems in the market.

\section{Assessment of serum levels of sex hormone- binding globulin}

SHBG is the blood transport protein (binding protein) that bind testosterone and estradiol.

The assay has been performed using SHBG ELISA.

Product: SHBG ELISA.

Type: Sandwich ELISA kit.

Sample type: Serum. 
Test principle: sandwich immunoassay.

\section{Assay procedure: First incubation (9 min)}

A measure of $10 \mu \mathrm{l}$ of the sample was incubated with both biotinylated, monoclonal SHBG-specific antibody and ruthenylated, monoclonal SHBG-specific antibody to form a sandwich complex.

\section{Second incubation (9 min)}

Streptavidin-coated microparticles were added to the reaction mixture and the complex binds to the solid phase via biotin-streptavidin interactions.

\section{Measurement}

The reaction mixture was transferred to a measuring cell and the microparticles were magnetically captured onto the surface of an electrode; the unbound sample was washed away before a chemiluminescent reaction was induced by applying a voltage to the electrode. Chemiluminescence was measured by a photomultiplier and the concentration of SHBG within the sample was calculated using acalibration curve.

Analysis of data was carried out using the SPSS software (the Statistical Package for the Social Sciences (version 21) (SPSS Inc., Chicago, Illinois, USA).

\section{Descriptive statistics}

Description of quantitative variables were given as mean, $\mathrm{SD}$, and range.

\section{Analytical statistics}

(1) The Wilcoxontest was used to compare the significance between the nonparametric data.

(2) The paired sample t-test was used to compare pretreatment and post-treatment values for hormonal and biochemical data with homogenic variability.

(3) $P$ values less than 0.05 were considered significant and $P$ values less than 0.01 were considered highly significant.

\section{Ethical approval/patient consent}

Institutional Review Board was provided by the Institutional Review Board for Faculty of Medicine, Suez Canal University, and Ismailia, Egypt. Written patient consent was taken from each patient.

\section{RESULTS}

This study was conducted on 113 male patients whose age ranged from 16 to 29 years $(21 \pm 2.95)$.

Table 1 shows that the mean number of Lehmann index was $(88.700 \pm 35.047)$ before treatment with ISO, and after treatment (6 months), there was areduction in Lehmann index number $(27.800 \pm 17.943)$, which was a statistically significant reduction of Lehmann index according to Wilcoxon test $(P<0.001)$.

Table 2 shows that pretreatment with ISO, the mean total testosterone serum level was $5.553 \pm 2.614$ and after treatment, there was a reduction in the mean total testosterone serum level $(3.329 \pm 2.276)$ with percentage of reduction $(34.54 \%)$, which was statistically significant reduction of mean total testosterone according to paired sample test $(P<0.005)$

Table 3 shows that pretreatment with ISO, the mean free testosterone serum level was $31.237 \pm 11.994$ and after treatment, there was reduction in mean free testosterone serum level (24.526 \pm 14.917$)$, with percentage of reduction of $33.46 \%$, which was statistically nonsignificant according toWilcoxon test $(P>0.05)$.

Table 4 shows that pretreatment with ISO, the mean SHBG serum level was $104.367 \pm 14.689$ and after treatment, there was elevation in mean SHBG serum level (105.533 \pm 16.362$)$,with percentage of elevation $(1.09 \%)$ which was statistically nonsignificant according toWilcoxon test $(P>0.05)$.

Table 1: Comparison of mean Lehmann index number before and after treatment with isotretinoin $(n=113)$

\begin{tabular}{|c|c|c|c|c|c|c|c|}
\hline \multirow[b]{2}{*}{ Lehmann index } & \multicolumn{2}{|c|}{ Time } & \multicolumn{2}{|c|}{ Paired differences } & \multirow[b]{2}{*}{$\%$ of change } & \multicolumn{2}{|c|}{ Wilcoxon test } \\
\hline & Pretreatment & Post-treatment & Mean & SD & & $\mathrm{Z}$ & $P$ value \\
\hline Range & $35-135$ & $5-90$ & & & & & \\
\hline Mean \pm SD & $88.700 \pm 35.047$ & $27.800 \pm 17.943$ & 60.900 & 28.192 & -70 & 8.29 & $<0.001^{*}$ \\
\hline Median & 85.05 & 25.5 & - & - & - & & \\
\hline
\end{tabular}


Table 2: Comparison of the mean total testosterone serum level pretreatment and post-treatment with isotretinoin ( $\mathrm{n}=113$ )

\begin{tabular}{|c|c|c|c|c|c|c|c|}
\hline \multirow[b]{2}{*}{$\begin{array}{l}\text { Total testosterone } \\
(\mathrm{ng} / \mathrm{ml})\end{array}$} & \multicolumn{2}{|c|}{ Time } & \multicolumn{2}{|c|}{ Paired differences } & \multirow[b]{2}{*}{$\%$ of change } & \multicolumn{2}{|c|}{ Wilcoxon test } \\
\hline & Pretreatment & Post-treatment & Mean & SD & & $t$ & $P$ value \\
\hline Range & $0.2-10.3$ & $0.2-8.6$ & 2.204 & 1.804 & -34.54 & 6.691 & $<0.001^{*}$ \\
\hline Mean \pm SD & $5.533 \pm 2.614$ & $3.329 \pm 2.276$ & & & & & \\
\hline
\end{tabular}

Table 3: Comparison of the mean free testosterone serum level pretreatment and post-treatment with isotretinoin( $\mathrm{n}=113$ )

\begin{tabular}{|c|c|c|c|c|c|c|c|}
\hline \multirow[b]{2}{*}{$\begin{array}{l}\text { Free testosterone } \\
(\mathrm{ng} / \mathrm{ml})\end{array}$} & \multicolumn{2}{|c|}{ Time } & \multicolumn{2}{|c|}{ Paired differences } & \multirow[b]{2}{*}{$\%$ of change } & \multicolumn{2}{|c|}{ Wilcoxon test } \\
\hline & Pretreatment & Post-treatment & Mean & $\mathrm{SD}$ & & $t$ & $P$ value \\
\hline Range & $7.9-56.9$ & $2.12-48.3$ & & & -34.54 & 6.691 & $<0.001^{*}$ \\
\hline Mean \pm SD & $31.237 \pm 11.994$ & $24.526 \pm 14.917$ & 6.710 & 20.548 & -33.46 & 1.89 & 0.074 \\
\hline Median & 30.51 & 25.21 & & & & & \\
\hline
\end{tabular}

Table 4: Comparison of the mean sex hormone-binding globulin serum level pretreatment and post-treatment with isotretinoin ( $\mathrm{n}=113$ )

\begin{tabular}{|c|c|c|c|c|c|c|c|}
\hline \multirow[b]{2}{*}{ SHBG $(\mu \mathrm{mol} / 1)$} & \multicolumn{2}{|c|}{ Time } & \multicolumn{2}{|c|}{ Paired differences } & \multirow[b]{2}{*}{$\%$ of change } & \multicolumn{2}{|c|}{ Wilcoxon test } \\
\hline & Pretreatment & Post-treatment & Mean & $\mathrm{SD}$ & & $Z$ & $P$ value \\
\hline Range & $80-150$ & $80-160$ & & & & & \\
\hline Mean \pm SD & $104.367 \pm 14.689$ & $105.533 \pm 16.362$ & -1.167 & 3.505 & 1.09 & -1.92 & 0.068 \\
\hline Median & 103.41 & 104.33 & & & & & \\
\hline
\end{tabular}

SHBG, sex hormone-binding globulin.

\section{DISCUSSION}

Acne vulgaris is a common skin disease involving blockage and inflammation of pilosebaceous units (hair follicle and their accompanying sebaceous gland) ${ }^{[4]}$. There are four processes that contribute to the development of acne: increased sebaceous gland activity, proliferation of P. acnes, and increased cell adhesion leading to comedone formation and inflammation ${ }^{[11]}$. Acne is considered a chronic disease owing to its prolonged course, pattern of recurrence and relapse, and manifestations such as acute outbreaks or slow onset ${ }^{[12]}$.
ISO is the only systemic agent that targets all four processes of acne ${ }^{[13]}$. ISO is a synthetic vitamin A analogthat was approved by the Food and Drug Administration in 1982 in the USA for the treatment of recalcitrant, severe, and nodulocystic acne ${ }^{[14]}$. ISO inhibits sebaceous gland function, decreases keratinization, decreases P. acnes, and suppresses the inflammatory response; the sebaceous gland activity is decreased by $90 \%$ within 4 weeks of ISO use ${ }^{[14]}$. Reasons supporting oral ISO as a first-line treatment for severe acne include clinical effectiveness, prevention of scarring, and quick improvement of a patient's quality of life ${ }^{[15]}$. 
Serum total testosterone was significantly lower after treatment with ISO $(5.533 \pm 2.614$ to $3.3329 \pm 2.276)$ as $P$ less than 0.001 . This agrees with Karadag et al. ${ }^{[9]}$ who studied the effect of different doses of ISO on pituitary hormones among 105 acne vulgaris patients, who were treated for 3 months with $0.2-0.5 \mathrm{mg} / \mathrm{kg} /$ day of ISO. There was significant reduction of total testosterone in the previous study $(3.59 \pm 3.74$ to $2.63 \pm 2.29)$.

The current study also agreed with another study which was conducted to detect the effect of ISO on pituitary hormone levels in acne patients. The study was carried out on 47 patients with acne vulgaris (31women and16 men), who were treated with $0.5-0.75 \mathrm{mg} / \mathrm{kg} /$ day of ISO for 3 months. Total testosterone was significantly lowered after the treatment with ISO $(P<0.005)$ in a latter study ${ }^{[16]}$.

Such findings were in agreement with another study conducted in Finland to detect the effect of ISO, tetracycline, and circulating hormones in acne andit was done on 19 patients with acne vulgaris ( 8 women and 11 men), and were received $0.5 \mathrm{mg} / \mathrm{kg} /$ day of ISO for $3-8$ months. Total testosterone was significantly reduced after the treatment $(22.2 \pm 8.4$ to $18.3 \pm 4.5)$ in the previous study ${ }^{[17]}$.

In another study, Boudou et al. ${ }^{[18]}$ studied the effect of oral ISO treatment on skin androgen receptor levels in male acne patients. The study was done on six male patients with acne vulgaris aged 19-30 years and were treated for 3 months with $0.5 \mathrm{mg} / \mathrm{kg} /$ day of ISO. There was no significant change in serum total testosterone. The differences in results may be related to the small sample size and limited duration in the study of Boudou et al. ${ }^{[18]}$.

There was no statistically significant change in serum levels of free testosterone post-treatment as $P=0.084$. This finding is matched with previous studies by Karadag et $a l .{ }^{[9]}$ which reported no statistically significant change in serum levels of free testosterone $(P=0.338$ and 0.04 , respectively) in men treated with ISO.

This study showed that there was elevation in SHBG levels after treatment $(104.367 \pm 14.689$ to $105.533 \pm 16.362)$, but it was not statistically significant $(P=0.079)$. This finding coordinated with other studies which reported no significant change in serum SHBG levels ${ }^{[16,17]}$.

Karadag et al.$^{[9]}$ in Turkey worked on 105 acne patients from two different centers. They divided the patients into three groups: the first group received $0.5-1 \mathrm{mg} / \mathrm{kg} / \mathrm{day}$, the second $0.2-0.5 \mathrm{mg} / \mathrm{kg} / \mathrm{day}$, and the third group received intermittent $0.5-1 \mathrm{mg} / \mathrm{kg} / \mathrm{day}$. They found that SHBG levels was significantly higher $(P<0.0001)$ with both low and higher doses of the treatment with ISO, which agree with the current thesis but without significance.

From the present study and data analysis, it has been shown that serum total testosterone levels were significantly lower after 4 months of treatment with ISO. The decrease in testosterone levels may partly explain the effectiveness of ISO for treating acne. Androgens are important in the pathogenesis of acne because they enhance follicular keratosis and influence sebum production.

\section{CONCLUSION}

In conclusion, this study showed that ISO treatment led to significant reduction in serum levels of total testosterone, which may be beneficial for tackling the pathogenesis of acne, but may affect androgen functions in general which should be examined in another study.

\section{CONFLICT OF INTEREST}

There are no conflicts of interest.

\section{REFERENCES}

1. BellewS, ThiboutotD, Del RossoJQ. Pathogenesis of acne vulgaris: what's new, what's interesting and what may be clinically relevant?. J Drug Dermatol2011; 10:582-585.

2. EichenfieldLF, Del RossoJQ, ManciniAJ. Evolving perspectives on the etiology and pathogenesis of acne vulgaris. J Drugs Dermatol2015; 14:263-272.

3. Knutsen-LarsonS, DawsonAL, DunnickCA, DellavalleRP. Acne vulgaris: pathogenesis, treatment, and needs assessment. Dermatol Clin 2012; 30:99-106.

4. DawsonAL, DellavalleRP. Acne vulgaris. BMJ Clin Res2013; 346:f2634.

5. RahmanM, SikderAU, RashidMM, KhondkerL. Association of serum testosterone with acne vulgaris in women. Bangabandhu Sheikh Mujib Med Univ J2012; 5:1-5.

6. LeydenJJ, Del RossoJQ, BarumEW. The use of isotretinoin in the treatment of acne vulgaris clinical considerations and future directions. J ClinAesthetDermatol2014; 7(2 Suppl):S3-S21

7. AgarwalUS, BesarwalRK, BholaK. Oral isotretinoin in different dose regimens for acne vulgaris: a randomized comparative trial. Indian J DermatolVenereolLeprol2011; 77:688-694.

8. YildizgorenMT, RifaiogluEN, DemirkapiM. Isotretinoin treatment in patients with acne vulgaris: does it impact muscle strength, fatigue, and endurance?. Cutis2015; 96:33-36. 
9. KaradagAS, TakciZ, ErtugrulDT, BilgiliSG, BalahorogluR, TakirM. The effect of different doses of isotretinoin on pituitary hormones. Dermatology2015; 230:354-359.

10. LehmannHP, RobinsonKA, AndrewsJS, HollowayV, GoodmanSN. Acne therapy: a methodologic review. J Am Acad Dermatol 2002; 47:231-40.

11. DubeauMF, IacucciM, BeckPL. Drug-induced inflammatory bowel disease and IBD-like conditions. Inflamm Bowel Dis2013; 19:445-456.

12. GollnickHPM, FinlayAY, ShearN. Can we define acne as a chronic disease? If so, how and when?. Am J ClinDermatol2008; 9:279-284.

13. BremnerJD, ShearerKD, McCafferyPJ. Retinoic acid and affective disorders: the evidence for an association. J Clin Psychiatry2012; 73:37-50.

14. ErgunT, SeckinD, OzaydinN. Isotretinoin has no negative effect on attention, executive function and mood. J EurAcad Dermatology Venereol $2012 ; 26: 431-439$.

15. LumsdenKR, NelsonAM, DispenzaMC. Isotretinoin increases skin-surface levels of neutrophil gelatinaseassociated lipocalin in patients treated for severe acne. Br J Dermatol 2011; 165:302-310.

16. KaradagAS, ErtugrulDT, TutalE, AkinKO. Isotretinoin influences pituitary hormone levels in acne patients. ActaDermVenereol2011; 91:31-34.

17. RiittaPalatsiR, RuokonenA, OikarinenA. Isotretinoin, tetracycline and circulating hormones in acne. ActaDermVenereol1997; 77:394-396.

18. BoudouP, SolimanH, ChivotM, VexiauP, FietJ. Effect of oral isotretinoin treatment on skin androgen receptor levels in male acneic patients. J ClinEndocrinol Metab1995; 80:1158-1161. 